\title{
Highly Selective Synthesis of Catalytically Active Monodisperse Rhodium Nanocubes
}

\author{
Yawen Zhang, ${ }^{\ddagger}$ Michael E. Grass, John N. Kuhn, Feng Tao, Susan E. Habas, Wenyu Huang, Peidong \\ Yang, and Gabor A. Somorjai* \\ Department of Chemistry, University of California, Berkeley, CA 94720, and the Chemical and Materials Sciences \\ Divisions, Lawrence Berkeley National Laboratory, 1 Cyclotron Road, Berkeley, CA 94720. \\ $\$$ College of Chemistry and Molecular Engineering, Peking University, Beijing 100871, China. \\ RECEIVED DATE (automatically inserted by publisher); E-mail: somorjai@berkeley.edu.
}

Synthesis of monodisperse and shape-controlled colloidal inorganic nanocrystals (NCs) is of increasing scientific interest and technological significance. ${ }^{1-8}$ Recently, shape control of $\mathrm{Pt},{ }^{1 \mathrm{a}, 3}$ $\mathrm{Pd},{ }^{4} \mathrm{Ag},{ }^{5} \mathrm{Au},{ }^{6}$ and $\mathrm{Rh}^{7} \mathrm{NCs}$ has been obtained by tuning growth kinetics in various solution-phase approaches, including modified polyol methods, ${ }^{1,3 a, 3 b, 4,5 a, 6 a, 7 a}$ seeded growth by polyol reduction, ${ }^{7 \mathrm{~b}, \mathrm{c}}$ thermolysis of organometallics, ${ }^{7 \mathrm{~d}}$ and micelle techniques. ${ }^{3 \mathrm{c}, 5 \mathrm{~b}, 6 \mathrm{~b}}$ Control of reduction kinetics of the noble metal precursors and regulation of the relative growth rates of low-index planes (i.e. $\{100\}$ and $\{111\}$ ) via selective adsorption of selected chemical species are two keys for achieving shape modification of noble metal NCs. One application for noble metal NCs of welldefined shape is in understanding how NC faceting (determines which crystallographic planes are exposed) affects catalytic performance. $^{2}$

$\mathrm{Rh} \mathrm{NCs}$ are used in many catalytic reactions, including hydrogenation, ${ }^{8 \mathrm{a}}$ hydroformylation, ${ }^{8 \mathrm{~b}}$ hydrocarbonylation, ${ }^{8 \mathrm{c}}$ and combustion reactions. ${ }^{8 \mathrm{~d}}$ Shape manipulation of Rh NCs may be important in understanding how faceting on the nanoscale affects catalytic properties, but such control is challenging and there are fewer reports on the shape control of Rh NCs compared to other noble metals. Xia and coworkers obtained Rh multipods exhibiting interesting surface plasmonic properties by a polyol approach. $^{7 \mathrm{a}}$ The Somorjai and Tilley groups synthesized crystalline Rh multipods, cubes, horns and cuboctahedra, via polyol seeded growth. ${ }^{7 \mathrm{~b}, \mathrm{c}}$ Son and colleagues prepared catalytically active monodisperse oleylamine-capped tetrahedral $\mathrm{Rh}$ NCs for the hydrogenation of arenes via an organometallic route. ${ }^{7 \mathrm{~d}}$ More recently, the Somorjai group synthesized sizetunable monodisperse Rh NCs using a one-step polyol technique. ${ }^{9}$

In this Communication, we report the highly selective synthesis of catalytically active, monodisperse $\mathrm{Rh}$ nanocubes of $<10 \mathrm{~nm}$ by a seedless polyol method. In this approach, $\mathrm{Br}^{-}$ions from trimethyl(tetradecyl)ammonium bromide (TTAB) effectively stabilize the $\{100)$ faces of Rh NCs, and induce the evolution of nanocubes (Scheme 1).

For a typical synthesis, $0.2 \mathrm{mmol} \mathrm{RhCl}_{3}$ hydrate, $1 \mathrm{mmol}$ $\mathrm{TTAB}$, and $4 \mathrm{mmol}$ poly(vinylpyrrolidone) (PVP, $M \mathrm{w}=24,000$ ), were added to $20 \mathrm{ml}$ ethylene glycol at room temperature. The stock solution was heated to $80{ }^{\circ} \mathrm{C}$ and purged for $20 \mathrm{~min}$ while stirring, producing a dark brown solution. The flask was then heated to $185{ }^{\circ} \mathrm{C}$ and maintained at this temperature for $1.5 \mathrm{~h}$ under an $\mathrm{Ar}$ atmosphere. When the reaction was complete, an excess of acetone was added to the solution at room temperature to precipitate the nanocubes. The $\mathrm{Rh}$ nanocubes were separated by centrifugation and washed twice by precipitation/dissolution with ethanol/hexanes.
Scheme 1. Seedless polyol synthesis of Rh nanocubes.
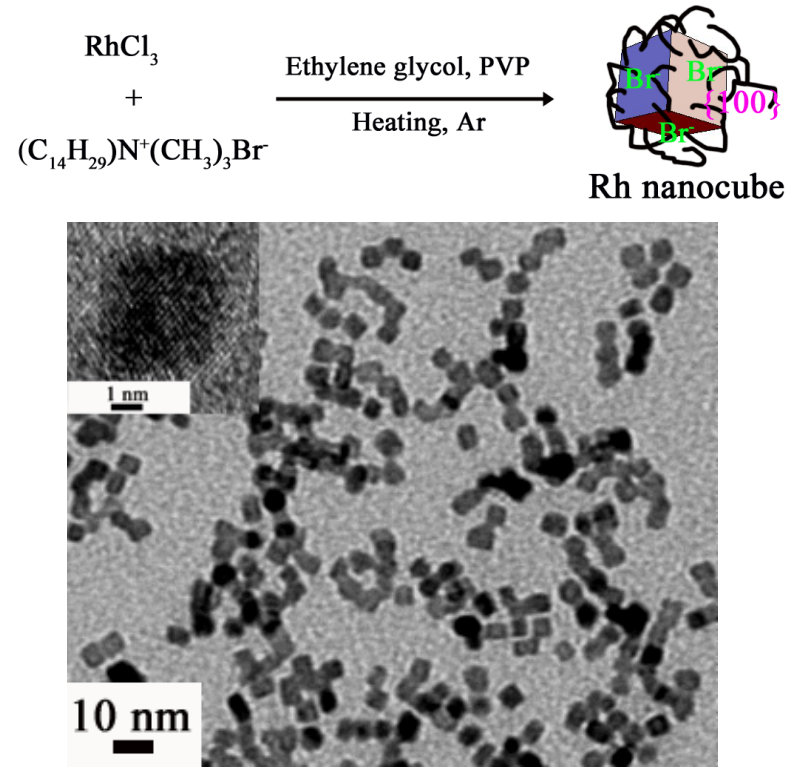

Figure 1. TEM and HRTEM (inset) images of Rh nanocubes.

Transmission electron microscopy (TEM, Philips FEI Tecnai $12,100 \mathrm{kV}$ ) revealed the formation of $6.4 \pm 0.5 \mathrm{~nm}$ (diagonal) $\mathrm{Rh}$ nanocubes with $>85 \%$ selectivity (Figures 1 and S1a). High resolution TEM (HRTEM, Philips CM200/FEG, 200 kV) (Figure 1 inset) indicated that the $\mathrm{Rh}$ nanocubes are single crystalline, enclosed by six $\{100\}$ faces. Energy dispersive X-ray (EDX) analysis of large areas of the TEM grid suggested that a significant fraction of the $\mathrm{Br}$ species were not removed by the precipitation/dissolution steps $(\mathrm{Br} / \mathrm{Rh}=0.41)$, while no $\mathrm{Cl}$ species were detectable (Figure S2). In addition, the EDX spectrum of a single $\mathrm{Rh}$ nanocube showed that a small fraction of $\mathrm{Br}$ species were associated with the nanocube $(\mathrm{Br} / \mathrm{Rh}=0.06)$, suggesting that TTAB interacts with the nanocube surfaces along with the PVP. X-ray diffraction (XRD, Bruker D8 GADDS, Co- $\mathrm{K}_{\alpha}$ radiation of $\lambda=1.79 \AA$ A) confirmed the formation of face-centered cubic $(f c c)$ $\mathrm{Rh}$ (Figure S3). The calculated lattice parameter was $a=0.3805$ $\mathrm{nm}$ for the Rh nanocubes (JCPDS: 0-685). The ratio of $I_{(111)} / I_{(200)}$ obtained for the Rh nanocubes is 2.44 , considerably lower than that (3.62-12.5) of Rh polygons of (111) orientation. ${ }^{9}$ X-ray photoelectron spectroscopy (XPS, Perkin-Elmer PHI 5300) demonstrated that the Rh NCs were composed of $\sim 76$ at $\% \mathrm{Rh}(0)$ and $\sim 24$ at $\% \mathrm{Rh}^{x+}$ (Figure S4). The intense $\mathrm{C} 1 s$ and $\mathrm{O} 1 s$ peaks and the weak $\mathrm{N} 1$ s peak, together with the barely detectable $\mathrm{Br} 3 d$ 
peak, revealed that PVP molecules were predominately adhered to the surface of the Rh nanocubes (Figure S4).

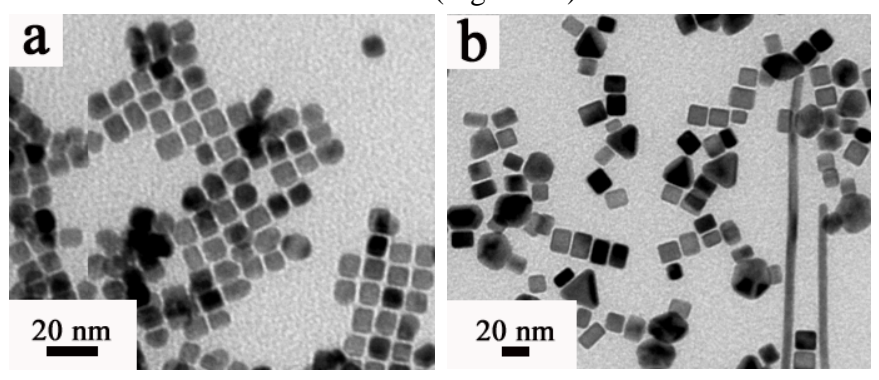

Figure 2. TEM images of (a) Pt and (b) Pd nanocubes.

Condition-dependent experiments were conducted to reveal the formation mechanism of the $\mathrm{Rh}$ nanocubes. In the absence of TTAB, the use of $\mathrm{RhCl}_{3}$ as the precursor produced faceted but polydisperse Rh NCs containing some tetrahedral particles (8.2 \pm $1.9 \mathrm{~nm}$; Figure S5a), while $\mathrm{RhBr}_{3}$ as the precursor yielded monodisperse, but not well-shaped cube-like NCs ( 40\% cubes) $\left(6.3 \pm 0.8 \mathrm{~nm}\right.$; Figure S5b). When a mix of $\mathrm{RhCl}_{3}$ and $\mathrm{RhBr}_{3}$ was adopted as the precursor, faceted but polydisperse cube-like NCs ( $39 \%$ cubes) were formed $(7.6 \pm 0.9 \mathrm{~nm}$; Figure S5c). Using $\mathrm{RhCl}_{3}$ as the precursor and TTAB as the $\mathrm{Br}^{-}$source, monodisperse $\mathrm{Rh}$ nanocubes with significantly enhanced selectivity (> 85\% cubes) and monodispersity were obtained with $\mathrm{Rh}^{3+} / \mathrm{TTAB}=1: 5$ $\left(6.4 \pm 0.5 \mathrm{~nm}\right.$; Figure 1). When $\mathrm{Rh}^{3+} / \mathrm{TTAB}=1: 1$, monodisperse Rh NCs dominated by truncated-cubes with exposed (100) and (111) facets, formed $(6.3 \pm 0.3 \mathrm{~nm}$; Figure S5d). These results strongly suggested that $\mathrm{Br}^{-}$ions from TTAB effectively stabilize the $\{100)$ facets of $\mathrm{Rh}$, and $\mathrm{Br}^{-} / \mathrm{Cl}^{-}$pairs regulate the relative growth rate along the $\langle 100\rangle$ and $\langle 111\rangle$ directions. ${ }^{4 c}$

$\mathrm{Pt}$ and $\mathrm{Pd}$ nanocubes can be also prepared by the same synthetic procedure. Reduction of $\left(\mathrm{NH}_{4}\right)_{2} \mathrm{PtCl}_{6}\left(\mathrm{Pt}^{4+} / \mathrm{TTAB}\right.$ $=1: 15)$ at $180{ }^{\circ} \mathrm{C}$ for $1 \mathrm{~h}$ produced $10.0 \pm 0.8 \mathrm{~nm}$ Pt nanocubes (74\% cubes, $20 \%$ cuboctahedra, $6 \%$ polyhedra) (Figures $2 \mathrm{a}$ and $\mathrm{S} 1 \mathrm{~b})$. Reduction of $\left(\mathrm{NH}_{4}\right)_{2} \mathrm{PdCl}_{6}\left(\mathrm{Pd}^{4+} / \mathrm{TTAB}=1: 15\right)$ at $140{ }^{\circ} \mathrm{C}$ for $1 \mathrm{~h}$ generated $17.4 \pm 3.2 \mathrm{~nm}$ Pd nanocubes (56\% cubes, $23 \%$ bars, $1 \%$ rods, and $20 \%$ polyhedra) (Figures $2 \mathrm{~b}$ and $\mathrm{S} 1 \mathrm{c}$ ). The calculated lattice constants were $a=0.3913 \mathrm{~nm}$ for the $\mathrm{Pt}$ nanocubes (JCPDS: 4-802), and $a=0.3867 \mathrm{~nm}$ for the Pd nanocubes (JCPDS: 46-1043) (Figure S3).
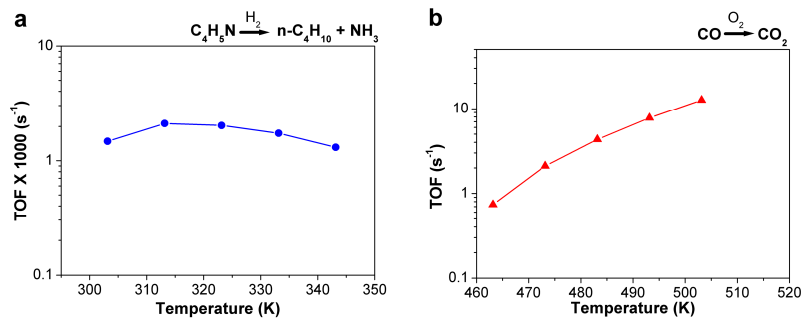

Figure 3. Turnover rate for (a) pyrrole hydrogenation to $n-\mathrm{C}_{4} \mathrm{H}_{10}$ and (b) $\mathrm{CO}$ oxidation to $\mathrm{CO}_{2}$ for $\mathrm{Rh}$ nanocubes as a function of temperature.

As-synthesized $\mathrm{Rh}$ nanocubes were deposited onto silicon wafers by the Langmuir-Blodgett (LB) technique to form 2dimensional nanoarray catalysts, ${ }^{9}$ which were then tested for pyrrole hydrogenation and $\mathrm{CO}$ oxidation. For pyrrole hydrogenation, the Rh catalysts fully hydrogenated pyrrole to $n$ butane and ammonia between 303 and $343 \mathrm{~K}$. The turnover frequency (TOF) did not strongly depend on temperature.
Detailed catalytic results on activity and selectivity will be presented for pyrrole hydrogenation in comparison with a $\mathrm{Rh}$ (100) single crystal elsewhere. For CO oxidation, the TOF monotonically increased from $0.74 \mathrm{~s}^{-1}$ at $463 \mathrm{~K}$ to $12.7 \mathrm{~s}^{-1}$ at 503 $\mathrm{K}$ (Figure 3b), and was comparable to those reported for a $\mathrm{Rh}$ (100) single crystal. ${ }^{10}$ The apparent activation energy for CO oxidation was $35 \mathrm{kcal} \mathrm{mol}^{-1}$, higher than that reported $(25.4 \mathrm{kcal}$ $\mathrm{mol}^{-1}$ ) for the $\mathrm{Rh}(100)$ single crystal. ${ }^{10}$

In conclusion, monodisperse sub-10 nm Rh nanocubes were synthesized with high selectivity by a seedless polyol method. The $\{100\}$ faces of the Rh NCs were effectively stabilized by chemically adsorbed $\mathrm{Br}^{-}$ions from TTAB. This simple one-step polyol route can be readily applied to the preparation of $\mathrm{Pt}$ and $\mathrm{Pd}$ nanocubes. Moreover, the organic molecules of PVP and TTAB that encapsulated the $\mathrm{Rh}$ nanocubes did not cause deactivation of the metal for catalytic turnover in pyrrole hydrogenation and $\mathrm{CO}$ oxidation. Currently, we are investigating the shape-dependent activity and selectivity of the Rh NCs for several heterogeneous reactions.

Acknowledgment. This work was supported by the Director, Office of Science, Office of Basic Energy Sciences, Division of Materials Sciences and Engineering of the U.S. Department of Energy under Contract No. DE-AC02-05CH11231 and portions of this work were performed at the Molecular Foundry, Lawrence Berkeley National Laboratory. Y.W.Z. appreciates the financial aid of Huaxin Distinguished Scholar Award from Peking University Education Foundation of China.

Supporting Information Available: More TEM images, size distribution histograms, XPS and EDX data, and detailed synthetic procedures are included in supporting information. This material is available free of charge via the Internet at http://pubs.acs.org.

* To whom correspondence should be addressed.

\section{References}

(1) (a) Ahmadi, T. S.; Wang, Z. L.; Green, T. C.; Henglein, A.; El-Sayed, M. A. Science 1996, 272, 1924. (b) Peng, X.; Manna, L.; Yang, W. Wickham, J.; Scher, E.; Kadavanich, A.; Alivisatos, A. P. Nature 2000 404, 59. (c) Sun, S.; Murray, C. B.; Weller, D.; Folks, L.; Moser, A Science 2000, 287, 1989. (d) Yin, Y.; Alivisatos, A. P. Nature 2005 437, 664. (e) Narayanaswamy, A.; Xu, H.; Pradhan, N.; Kim, M.; Peng, X. J. Am. Chem. Soc. 2006, 128, 10310. (f) Habas, S. E.; Lee, H.; Radmilovic, V.; Somorjai, G. A.; Yang, P. Nature Mater. 2007, 6, 692.

(2) (a) Narayanan, R.; El-Sayed, M. A. J. Phys. Chem. B 2005, 109, 12663. (b) Tian, N.; Zhou, Z. Y.; Sun, S. G.; Ding, Y.; Wang, Z. L. Science 2007, 316, 732.

(3) (a) Song, H.; Kim, F.; Connor, S.; Somorjai, G. A.; Yang, P. J. Phys Chem. B 2005, 109, 188. (b) Chen, J.; Herricks, T.; Xia, Y. Angew Chem. Int. Ed. 2005, 44, 2589. (c) Lee, H.; Habas, S. E.; Kweskin, S. Butcher, D.; Somorjai, G. A.; Yang, P. Angew. Chem. Int. Ed., 2006, 45 , 7824

(4) (a) Xiong, Y.; Chen, J.; Benjamin, J. W.; Xia, Y. J. Am. Chem. Soc. 2005, 127, 7332. (b) Xiong, Y., McLellan, J. M.; Yin, Y; Xia, Y Angew. Chem. Int. Ed. 2007, 46, 790. (c) Xiong, Y.; Cai, H.; Wiley, B. J.; Wang, J.; Kim, M. J.; Xia, Y. J. Am. Chem. Soc. 2007, 129, 3665.

(5) (a) Tao, A.; Sinsermsuksakul, P.; Yang, P. Angew. Chem. Int. Ed. 2006, 45, 4597. (b) Chen, S; Carroll, D. L. J. Phys. Chem. B 2004, 108, 5500.

(6) (a) Seo, D.; Park, J. C.; Song, H. J. Am. Chem. Soc. 2006, 128, 14863 (b) Wu, H.-Y.; Liu, M.; Huang, M. H. J. Phys. Chem. B 2006, 110 19291

(7) (a) Zettsu, N.; McLellan, J. M.; Wiley, B.; Yin, Y.; Li, Z.-Y.; Xia, Y. Angew. Chem. Int. Ed. 2006, 45, 1288. (b) Humphrey, S. M.; Grass, M E.; Habas, S. E.; Niesz, K.; Somorjai, G. A.; Tilley, T. D., Nano Lett. 2007, 7, 785 (c) Hoefelmeyer, J. D; Niesz, K. Somorjai, G. A. Tilley, T. D. Nano Lett. 2005, 5, 435. (d) Park, K. H.; Jang, K.; Kim, H. J.; Son, S. U. Angew. Chem. Int. Ed. 2007, 46,1152.

(8) (a) Pellegatta, J.-L.; Blandy, C.; Collière, V.; Choukroun, R.; Chaudret B.; Cheng P.; Philippot, K. J. Mol. Catal. A 2002, 178, 55. (b) Yoon, T.J.; Kim, J. I.; Lee J.-K. Inorg. Chim. Acta 2003, 345, 228. (c) Halttunen, M. E.; Niemelä, M. K.; Krause, A.O.I.; Vaara, T.; Vuori, A.I. Appl. Catal. A 2001, 205, 37. (d) Gayen, A.; Baidya, T.; Biswas, K.; Roy, S. Hegde, M.S. Appl. Catal. A 2006, 315, 135

(9) Zhang, Y.; Grass, M. E.; Habas, S. E.; Tao, F.; Zhang, T.; Yang, P. Somorjai, G. A. J. Phys. Chem. C 2007, 111, 12243.

(10) Peden, C. H. F.; Goodman, D. W.; Blair, D. S.; Berlowitz, P. J.; Fisher, G. B.; Oh, S. H. J. Phys. Chem. 1988, 92, 1563 


\section{$\mathrm{RhCl}_{3}$ \\ $+$ \\ $\left(\mathrm{C}_{14} \mathrm{H}_{29}\right) \mathrm{N}^{+}\left(\mathrm{CH}_{3}\right)_{3} \mathrm{Br}$ \\ Ethylene glycol, PVP \\ Heating, Ar

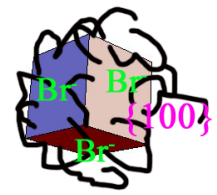 \\ $\mathrm{Rh}$ nanocube}

Monodisperse sub-10 nm Rh nanocubes were synthesized with high selectivity (>85\%) by a seedless polyol method. The $\{100\}$ faces of the Rh NCs were effectively stabilized by chemically adsorbed Br- ions from trimethyl(tetradecyl)ammonium bromide (TTAB). This simple one-step polyol route can be readily applied to the preparation of $\mathrm{Pt}$ and Pd nanocubes. Moreover, the organic molecules of PVP and TTAB that encapsulated the Rh nanocubes did not cause deactivation of the metal for catalytic turnover in pyrrole hydrogenation and $\mathrm{CO}$ oxidation. 